Check for updates

Cite this: Phys. Chem. Chem. Phys., 2017, 19, 17356

Received 6th April 2017,

Accepted 19th June 2017

DOI: $10.1039 / c 7 c p 02229 h$

rsc.li/pccp

\title{
High cross-plane thermoelectric performance of metallo-porphyrin molecular junctions $\dagger$
}

\author{
Mohammed Noori, $\ddagger^{\mathrm{ab}}$ Hatef Sadeghi, (D) $\ddagger^{*^{a}}$ Qusiy Al-Galiby, $\ddagger^{\text {ac }}$ Steven W. D. Bailey ${ }^{a}$ \\ and Colin J. Lambert $\left(\mathbb{D} \ddagger^{*^{a}}\right.$
}

\begin{abstract}
We investigated the thermoelectric properties of flat-stacked 5,15-diphenylporphyrins containing divalent metal ions $\mathrm{Ni}, \mathrm{Co}, \mathrm{Cu}$ or $\mathrm{Zn}$, which are strongly coordinated with the nitrogens of pyridyl coated gold electrodes. Changing metal atom has little effect on the thermal conductance due to the phonons. The room-temperature Seebeck coefficients of these junctions are rather high, ranging from $90 \mu \mathrm{V} \mathrm{K}{ }^{-1}$ for $\mathrm{Cu}, \mathrm{Ni}$ and $\mathrm{Zn}$-porphyrins to $-16 \mu \mathrm{V} \mathrm{K} \mathrm{K}^{-1}$ for Co-porphyrin. These values could be further increased by lowering molecular energy levels relative to the DFT-predicted Fermi energy. In contrast, the phonon contribution to the thermal conductance of these junctions is rather insensitive to the choice of metal atom. The thermopower, thermal conductance and electrical conductance combined to yield the room-temperature values for the thermoelectric figure of merit $Z T$ ranging from 1.6 for Cu porphyrin to $\sim 0.02$ for Ni-porphyrin.
\end{abstract}

\section{Introduction}

Single-molecule thermoelectricity is a rapidly expanding field of fundamental research, which will eventually inform the design and synthesis of high-performance thermoelectric molecular films. Early studies showed that factors governing the sign and magnitude of the Seebeck coefficient $S$ include the length of conjugated molecules and the type of terminal group. For examples thiol-terminated molecules typically exhibit HOMOdominated transport and a positive $S$, ranging from 6.8 to $2.4 \mu \mathrm{V} \mathrm{K}^{-1}$ for $n$-alkanedithiols, depending on the length $n,{ }^{1,2}$ from $7 \mu \mathrm{V} \mathrm{K}^{-1}$ to $16 \mu \mathrm{V} \mathrm{K}{ }^{-1}$ for $n$-benzenedithiols, depending on the number $n$ of phenyl rings, ranging from $7 \mu \mathrm{V} \mathrm{K} \mathrm{K}^{-1}$ to $16 \mu \mathrm{V} \mathrm{K}^{-1} \cdot{ }^{3-9}$ Positive Seebeck coefficients up to $24 \mu \mathrm{V} \mathrm{K} \mathrm{K}^{-1}$ were measured for 1,4-bis((trimethylstannyl)methyl)- $n$-phenyl $(n=1,2,3,4)$, up to $10.4 \mu \mathrm{V} \mathrm{K} \mathrm{K}^{-11,10,11}$ for 1,4-n-phenylenediamine (PDA) $(n-1,2,3)$ and as high as $14.8 \mu \mathrm{V} \mathrm{K} \mathrm{K}^{-1}$ for oligothiophenes on gold. ${ }^{12}$ On the other hand pyridyl terminal groups promote LUMO-dominated transport leading to negative Seebeck coefficients as high as $-9 \mu \mathrm{V} \mathrm{K}{ }^{-1}$ and $-10 \mu \mathrm{V} \mathrm{K} \mathrm{K}^{-1}$ respectively ${ }^{11,13} 4,4^{\prime}$-for bipyridine and 1,2-di(4-pyridyl)ethylene respectively. Fullerenes also exhibit negative Seebeck coefficients,

\footnotetext{
a Quantum Technology Centre, Department of Physics, Lancaster University, Lancaster, LA1 4YB, UK. E-mail: h.sadeghi@lancaster.ac.uk, c.lambert@lancaster.ac.uk

${ }^{b}$ Department of Physics, Collage of Science, Thi-Qar University, Thi-Qar, Iraq

${ }^{c}$ Department of Physics, Collage of Education, Al Qadisiyah University, Iraq

$\dagger$ Electronic supplementary information (ESI) available. See DOI: 10.1039/ c7cp02229h

\$ These author contributed equally to this work.
}

ranging from -10 to $-30 \mu \mathrm{V} \mathrm{K}{ }^{-1}$ for $\mathrm{C}_{60}{ }^{14}$ to $-33 \mu \mathrm{V} \mathrm{K}{ }^{-1}$ for $\mathrm{C}_{60}$ dimers ${ }^{15}$ and up to $-31.6 \mu \mathrm{V} \mathrm{K}^{-1} \mathrm{C}_{82}$ endohedral fullerenes. ${ }^{16}$ The sign of the endohedral fullerene $\mathrm{Sc} 3 \mathrm{~N} @ \mathrm{C}_{80}$ was shown to be sensitive to pressure, ranging from $-25 \mu \mathrm{V} \mathrm{K}{ }^{-1}$ to $+25 \mu \mathrm{V} \mathrm{K}^{-1}$, depending on the orientation of the molecule on a gold substrate. ${ }^{17}$ Other factors predicted to govern the thermoelectric performance of molecules include the type of electrode. Although most of the above studies used gold electrodes, alternative choices could include graphene, ${ }^{18,19}$ silicene, ${ }^{20,21}$ platinum, ${ }^{22}$ palladium, ${ }^{22}$ iron $^{23}$ and nickel, ${ }^{24}$ which provide a range of Debye frequencies for controlling phonon transport across the electrode-molecule boundary. $^{24}$

Here, in an effort to further enhance the thermoelectric performance of single-molecule junctions by controlling phonon transport while maintaining good electrical contact, we examine the effect of coating the electrodes with functional units, which form coordination bonds with the molecule of interest. As an example, we examine junctions formed from 5,15-diphenylporphyrins containing divalent metal ions $\mathrm{Ni}, \mathrm{Co}, \mathrm{Cu}$ or $\mathrm{Zn}$, which are strongly coordinated with the nitrogens of pyridyl coated gold electrodes. Porphyrins are attractive as building blocks for molecular-scale devices, because they are conjugated, rigid, chemically stable molecules and their properties could be modified by coordinating a variety of metallic ions (metallo-porphyrins). ${ }^{25-33}$ In what follows our aim is to explore the potential of the metallo-porphyrin-based molecular junctions shown in Fig. 1a for high-efficiency thermoelectricity. Since the in-plane electrical conductance of porphyrins is rather low, we consider "cross-plane" junctions in which the current flows perpendicular to the porphyrin plane. ${ }^{34}$ A shown in Fig. 1b, a porphyrin plane is contacted to gold electrodes via pyridyl 
(a)

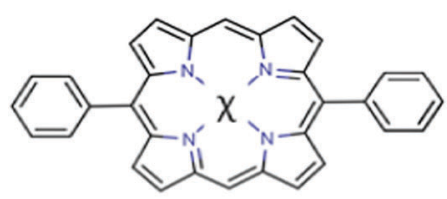

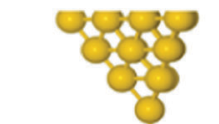

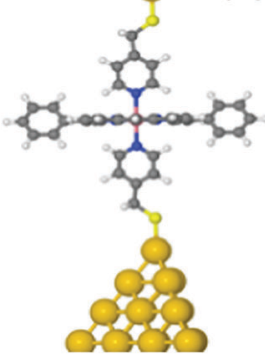

Fig. 1 Diphenylporphyrins-based molecular structures (a) with metallic atom, (b) an example of an optimized configuration of the system containing a single molecule $\chi$-DPP sandwiched between two gold leads which in turn are activated by pyridine-4-yl-methanethiol with different metallic atoms, where $\chi=\mathrm{Co}, \mathrm{Cu}, \mathrm{Ni}$ or $\mathrm{Zn}$.

groups attached to the gold by thiol anchors, with the plane of the porphyrin stacked perpendicular to the direction of charge transport.

From the point of view of optimising thermoelectric properties, junctions formed from these molecules are of interest, because by varying the metal atom (denoted $\chi$ in Fig. 1) residing in the core of the organic porphyrin framework, the molecular energy levels can be tuned relative to the Fermi energy $E_{\mathrm{F}}$ of the electrodes and if energy levels are close enough to $E_{\mathrm{F}}$, then this should lead to transport resonances, which enhance the thermopower. ${ }^{35}$ In what follows, we shall demonstrate that this is indeed the case and that large positive and negative thermopowers are achievable. Moreover, our calculations show that although the thermal conductance due to the phonons dominates the total thermal conductance, high values of $Z T$ are obtained.

Recent experiments and theory show that the conductances $G$ of the metallo-porphyrins are enhanced when they are sandwiched between two gold electrodes with pyridine-4-yl-methanethiol anchors (Fig. 1b) ${ }^{34,36}$ Since large $G$ is desirable, the 5,15-diphenylporphyrins $(\chi$-DPP) studied in this paper contains metal atoms $\chi=\mathrm{Co}, \mathrm{Cu}$, $\mathrm{Ni}$ or Zn, which are strongly coordinated with the nitrogens of pyridyl coated gold electrodes (Fig. 1a). Our aim in this paper is to investigate the thermoelectric properties of these metalloporphyrins devices when the metal atom is varied.

\section{Methods}

To calculate the electronic and vibrational properties of each metallo-porphyrin, we used the spin density functional (DFT) code SIESTA, ${ }^{37}$ which employs Troullier-Martins pseudopotentials to represent the potentials of the atomic cores, ${ }^{38}$ and a local atomicorbital basis set. We used a double-zeta polarized basis set ${ }^{39}$ for all atoms and the local density functional approximation (LDA-CA) by Ceperley and Adler. ${ }^{40}$ The Hamiltonian and overlap matrices are calculated on a real-space grid defined by a plane-wave cutoff of 150 Ry. Each molecule was relaxed to the optimum geometry until the forces on the atoms are smaller than $0.02 \mathrm{eV}^{-1}$. The only periodicity is in the electrodes, which are assumed to be composed of a periodic series of principal layers (see Fig. S10, ESI $\dagger$ ). In the transverse direction, there is no periodicity and the unit cell is sufficiently large that there is no interaction between the molecule and its image. The mean field Hamiltonian obtained using DFT describes the whole structure including the leads, the interface between the leads and molecule and molecule itself. In this way, all parts of the structure are treated in a holistic manner, at the same level of DFT.

\section{Results and discussion}

To obtain the optimized geometry (and the associated mean field Hamiltonian) of $\chi$-DPP we employed the SIESTA ${ }^{37}$ implementation of spin-polarized density functional theory (DFT) and from the converged mean field DFT Hamiltonian, we used our quantum transport code, Gollum ${ }^{41}$ to compute the electronic transmission coefficient $T_{\mathrm{el}}^{\sigma}(E)$ for electrons with energy $E$ and spin $\sigma=[\uparrow, \downarrow]$ passing from one electrode to the other via the $\chi$-DPP. For each $\chi$-DPP in Fig. $1 \mathrm{~b}$ and $2 \mathrm{a}-\mathrm{d}$ shows total electronic transmission coefficients as a function of energy for $\chi=\mathrm{Co}, \mathrm{Cu}, \mathrm{Zn}$ or $\mathrm{Ni}$. The corresponding spin-dependent transmissions $T_{\mathrm{el}}^{\sigma}(E)$ are shown in the ESI. $\dagger$

Due to the presence of thiol anchors, the DFT-predicted Fermi energy is close to the HOMO transmission resonances of each $\chi$-DPP and the slope of $T_{\mathrm{el}}^{\sigma}\left(E_{\mathrm{F}}\right)$ is negative. Hence from eqn (S5) of the ESI, $\dagger$ one expects the Seebeck coefficient $S$ to be positive. Using the Landauer formula (see ESI $\dagger$ ), the electrical conductance was calculated from the transmission coefficient shown in Fig. 2.

The electrical conductances (Fig. S2 of the ESI $\dagger$ ) follow the trend $G_{\mathrm{Zn}}>G_{\mathrm{Cu}}>G_{\mathrm{Co}}>G_{\mathrm{Ni}}$ in agreement with the experimental values of $\mathrm{Zn}$-DPP $8.2 \times 10^{-2}$, Cu-DPP $4.4 \times 10^{-2}$, Co-DPP $2.2 \times 10^{-2}$ and Ni-DPP $1.8 \times 10^{-2} .^{33}$

To calculate the vibrational modes of each structure, we use the harmonic approximation to construct the dynamical matrix D and a Green's function approach to calculate the phononic
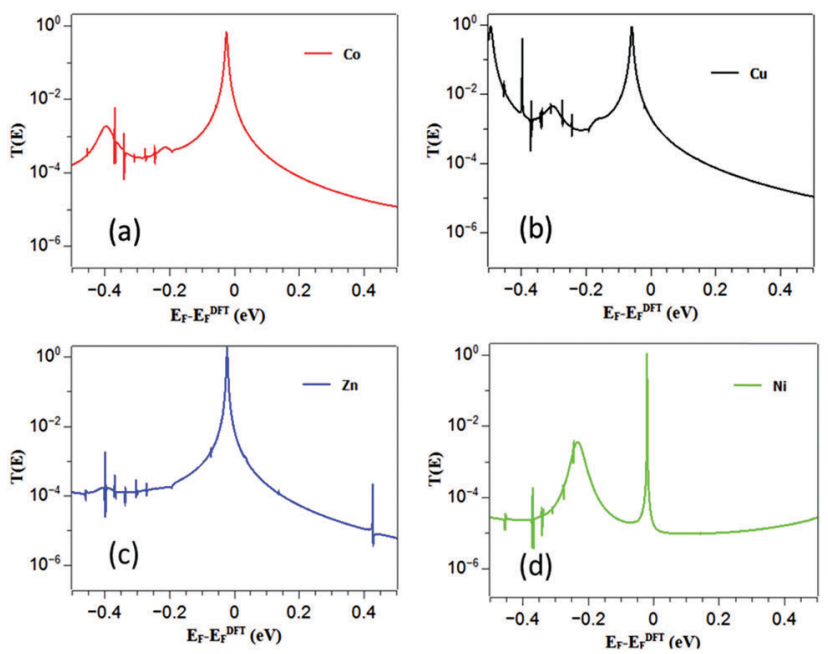

Fig. $2(a-d)$ show the electronic transmission coefficients $T(E)=T_{\mathrm{el}}^{\uparrow}(E)+T_{\mathrm{el}}^{\downarrow}(E)$ as a function of energy $E$ for $\chi$-DPP with $\chi=\mathrm{Co}, \mathrm{Cu}, \mathrm{Zn}$ and $\mathrm{Ni}$. 

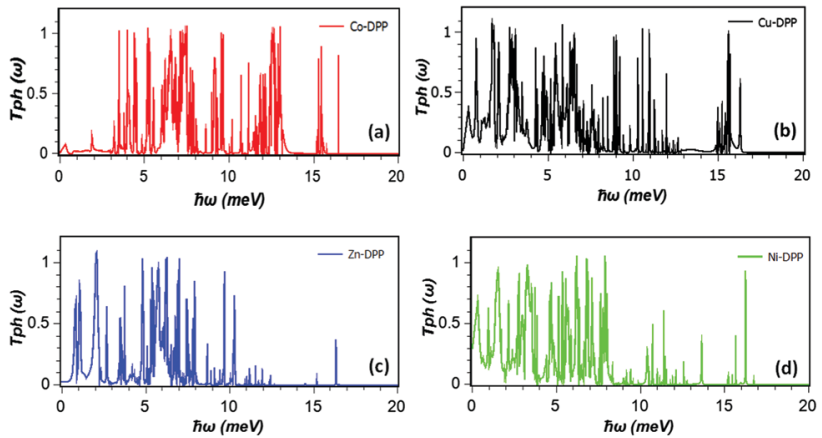

Fig. $3(a-d)$ Phonon transmission coefficient as a function of $\hbar \omega$ for $\chi$-DPP with $\chi=\mathrm{Co}, \mathrm{Cu}, \mathrm{Zn}$ and $\mathrm{Ni}$.

transmission coefficient $T_{\mathrm{ph}}(\omega)$ for phonons with energy $\hbar \omega$ passing through the device as explained in ref. 35. Fig. 3 shows the phonon transmission coefficients for each $\chi$-DPP.

The thermal conductance of the junction $\left(\kappa=\kappa_{\mathrm{ph}}+\kappa_{\mathrm{el}}\right)$ is obtained by summing the contributions from both electrons $\left(\kappa_{\mathrm{el}}\right)$ and phonons $\left(\kappa_{\mathrm{ph}}\right)$. The electronic (phononic) thermal conductances are calculated from the electronic (phononic) transmission coefficients (as described in ref. 35).

Comparison between Fig. $4 \mathrm{a}$ and $\mathrm{b}$ shows that in the vicinity of DFT Fermi energy, the main source of the thermal conductance in these junctions is due to phonons, whose room-temperature contribution is relatively insensitive to the choice of metal atom, varying between 6 and $10 \mathrm{pW} \mathrm{K}^{-1}$ depending on the choice. The inset of Fig. $4 \mathrm{~b}$ shows the cumulative distribution of eigenvalues (integrated density of states) of each molecule and reveals that the lower frequency modes of the Co porphyrin in the region 0 to $1.5 \mathrm{meV}$ are pushed to higher frequencies, leading to a
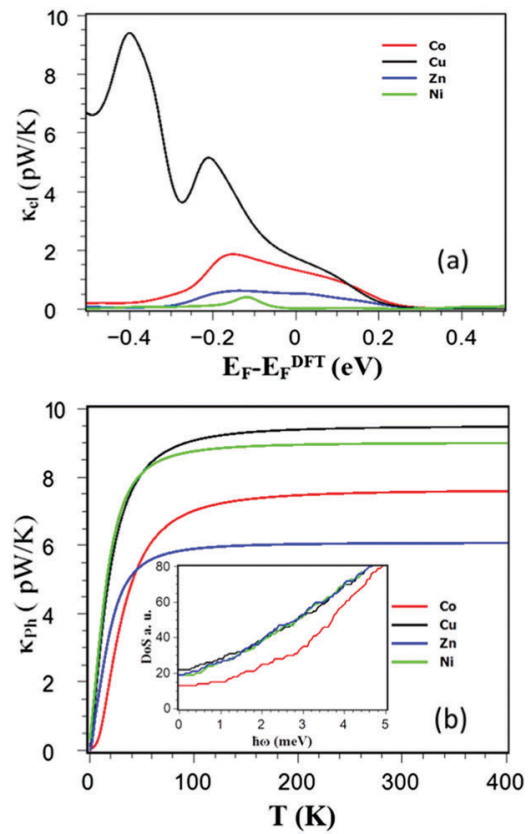

Fig. 4 (a) Show electronic contribution of the thermal conductance $\kappa_{\mathrm{el}}$ and (b) show phononic contribution of the thermal conductance $\kappa_{\text {ph }}$ for DPP with Co, $\mathrm{Cu}, \mathrm{Zn}$ and $\mathrm{Ni}$. low-frequency transmission gap below $3 \mathrm{meV}$. This reflects the higher binding energy of a single pyridyl group to the Co metal atom compared with the other. (These follow the trend $\mathrm{Co}=-1.2 \mathrm{eV}, \mathrm{Zn}=-1.2 \mathrm{eV}, \mathrm{Cu}=-0.4 \mathrm{eV}$ and $\mathrm{Ni}=-0.17 \mathrm{eV}$.) (These follow the trend $\mathrm{Co}=-1.2 \mathrm{eV}, \mathrm{Zn}=-1.2 \mathrm{eV}, \mathrm{Cu}=-0.4 \mathrm{eV}$ and $\mathrm{Ni}=-0.17 \mathrm{eV}$.)

Consequently the low-frequency cumulative distribution of Co and the low-temperature thermal conductance initially lies below that of the others and then rises with a steeper slope. Fig. 3c shows that $\mathrm{Zn}$ also has a low-frequency transmission gap below $1 \mathrm{meV}$, which suppresses the low-temperature phonon thermal conductance.

Fig. 5 shows the Seebeck coefficient $S$ and total figure of merit $Z T$ over a range of Fermi energies at room temperature for each metallo-porphyrin. Fig. 5a demonstrates that both the magnitude and sign of $S$ are sensitive to the metal atom at the centre of the DPP, which determine the location of transport resonances relative to the Fermi energy. Since the Fermi energy from DFT is not necessarily reliable, we have presented results for a range of Fermi energies (Fig. 5a).

For the junctions discussed in this paper, the conductances have been measured experimentally ${ }^{36}$ so we have estimated the Fermi energy by comparing these measurements with our calculated conductances. This yields a value of $E_{\mathrm{F}}=-0.03 \mathrm{eV}$, compared with the DFT-predicted Fermi energy. Table 1 shows the Seebeck coefficients of porphyrins in the presence of $\mathrm{Cu}$, and then $\mathrm{Co}, \mathrm{Zn}$ and $\mathrm{Ni}$ at $E_{\mathrm{F}}=-0.03 \mathrm{eV}$. The predicted trend is $S_{\mathrm{Cu}}>S_{\mathrm{Ni}}>S_{\mathrm{Zn}}>S_{\mathrm{Co}}$ and when combined with the higher $\mathrm{Cu} \mathrm{CPP}$ the yield room-temperature values of $Z T$ range from 1.66 for $\mathrm{Cu}$ porphyrin to 0.07 for $\mathrm{Zn}$ porphyrin, as shown in Fig. 5b.
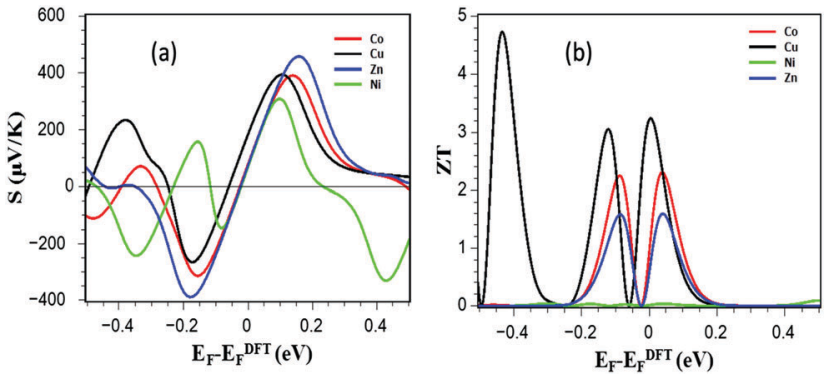

Fig. 5 Show (a) Seebeck coefficient $S$ (thermopower) and (b) total figure of merit $Z T$ over a range of Fermi energies at room temperature for each metallo-porphyrin.

Table 1 Seebeck coefficient $S$ (thermopower) and total figure of merit $Z T$ at $E_{\mathrm{F}}-E_{\mathrm{F}}^{\mathrm{DFT}}=-0.03 \mathrm{eV}^{33}$

\begin{tabular}{llllll}
\hline & $S\left(\mu \mathrm{V} \mathrm{K}^{-1}\right)$ & & $Z T$ & \\
\cline { 2 - 3 } \cline { 5 - 6 } Metallic & $E_{\mathrm{F}}=-0.03 \mathrm{eV}$ & $E_{\mathrm{F}}=0.0 \mathrm{eV}$ & & $E_{\mathrm{F}}=-0.03 \mathrm{eV}$ & $E_{\mathrm{F}}=0.0 \mathrm{eV}$ \\
\hline $\mathrm{Cu}$ & +90 & +182 & 1.66 & 3.22 \\
$\mathrm{Co}$ & -16 & +77 & 0.05 & 0.92 \\
$\mathrm{Zn}$ & -23 & +72 & 0.07 & 0.56 \\
$\mathrm{Ni}$ & -32 & +66 & 0.018 & 0.05
\end{tabular}




\section{Conclusion}

The room-temperature Seebeck coefficients of these junctions are rather high, ranging from $90 \mu \mathrm{V} \mathrm{K}^{-1}$ for $\mathrm{Cu}$-porphyrin to $-16 \mu \mathrm{V} \mathrm{K}^{-1}$ for Co-porphyrin. The corresponding room-temperature values of $Z T$ range from 1.66 for $\mathrm{Cu}$ porphyrin to $\sim 0.02$ for $\mathrm{Ni}$ porphyrin. These values could be further increased by shifting molecular energy levels relative to the Fermi, either by doping or gating, which moves the Fermi energy closer to the regions of higher thermopower in Fig. 5. These results demonstrate that metalloporphyrins are attractive building blocks for molecular-scale thermoelectricity and by passing thermal and charge currents perpendicular to the plane of the porphyrins, large values of the Seebeck coefficient and figure of merit are possible.

\section{Acknowledgements}

This work is supported by UK EPSRC grants EP N017188/1, EP/M014452/1, the European Union Marie-Curie Network MOLESCO 606728 and the Ministry of Higher Education and Scientific Research, Thi-Qar University, IRAQ.

\section{References}

1 J. A. Malen, et al., Nano Lett., 2009, 9, 1164.

2 S. Y. Guo, G. Zhou and N. J. Tao, Nano Lett., 2013, 13, 4326.

3 A. Tan, S. Sadat and P. Reddy, Appl. Phys. Lett., 2010, 96, 013110.

4 A. Tan, et al., J. Am. Chem. Soc., 2011, 133, 8838.

5 S. K. Lee, et al., Nano Lett., 2014, 14, 5276.

6 Y. Kim, et al., Appl. Phys. Lett., 2016, 109, 033102.

7 M. Tsutsui, M. Taniguchi and T. Kawai, Nano Lett., 2008, 8, 3293.

8 T. Morikawa, et al., Nanoscale, 2014, 6, 8235.

9 S. Kaneko, et al., Appl. Phys. Express, 2015, 8, 095503.

10 D. Kim, P. S. Yoo and T. Kim, J. Korean Phys. Soc., 2015, 66, 602.

11 T. Kim, et al., Nano Lett., 2014, 14, 794.

12 W. B. Chang, et al., Chem. Mater., 2014, 26, 7229.

13 J. R. Widawsky, et al., Nano Lett., 2012, 12, 354.

14 S. K. Yee, et al., Nano Lett., 2011, 11, 4089.

15 C. Evangeli, et al., Nano Lett., 2013, 13, 2141.

16 S. K. Lee, et al., Nanoscale, 2015, 7, 20497.

17 L. Rincon-Garcia, et al., Nat. Mater., 2016, 15, 289.

18 H. Sadeghi, J. A. Mol, C. S. Lau, G. A. D. Briggs, J. Warner and C. J. Lambert, Proc. Natl. Acad. Sci. U. S. A., 2015, 112, 2658-2663.

19 C. S. Lau, et al., Nano Lett., 2016, 16, 170-176.

20 H. Sadeghi, S. Bailey and C. J. Lambert, Appl. Phys. Lett., 2014, 104(10), 103104.
21 H. Sadeghi, S. Sangtarash and C. J. Lambert, Sci. Rep., 2015, $5,9514$.

22 V. M. García-Suárez, A. R. Rocha, S. W. Bailey, C. J. Lambert, S. Sanvito and J. Ferrer, Phys. Rev. B: Condens. Matter Mater. Phys., 2005, 72, 045437.

23 V. M. García-Suárez, C. M. Newman, C. J. Lambert, J. M. Pruneda and J. Ferrer, J. Phys.: Condens. Matter, 2004, 16, 5453.

24 H. Sadeghi, S. Sangtarash and C. J. Lambert, Nano Lett., 2015, 15, 7467-7472.

25 G. Dorough, J. Miller and F. M. Huennekens, J. Am. Chem. Soc., 1951, 73, 4315-4320.

26 G. Sedghi, V. M. García-Suárez, L. J. Esdaile, H. L. Anderson, C. J. Lambert, S. Martín, D. Bethell, S. J. Higgins, M. Elliott and N. Bennett, Nat. Nanotechnol., 2011, 6, 517-523.

27 D. Gust and J. D. Roberts, J. Am. Chem. Soc., 1977, 99, 3637-3640.

28 Z. Li, M. Smeu, M. A. Ratner and E. Borguet, J. Phys. Chem. C, 2013, 117, 14890-14898.

29 Z. Li and E. Borguet, J. Am. Chem. Soc., 2011, 134, 63-66.

30 Z.-F. Liu, S. Wei, H. Yoon, O. Adak, I. Ponce, Y. Jiang, W.-D. Jang, L. M. Campos, L. Venkataraman and J. B. Neaton, Nano Lett., 2014, 14, 5365-5370.

31 Y. Li, J. Yao, S. Zhong and Z. Zou, Curr. Appl. Phys., 2011, 11, 1349-1353.

32 R. Ferradás, V. M. García-Suárez and J. Ferrer, 2012, arXiv preprint arXiv:1208.3157.

33 I. Beletskaya, V. S. Tyurin, A. Y. Tsivadze, R. Guilard and C. Stern, Chem. Rev., 2009, 109, 1659-1713.

34 A. C. Aragonès, N. Darwish, W. J. Saletra, L. Pérez-García, F. Sanz, J. Puigmartí-Luis, D. B. Amabilino and I. Díez-Pérez, Nano Lett., 2014, 14, 4751-4756.

35 H. Sadeghi, S. Sangtarash and C. J. Lambert, Nano Lett., 2015, 15, 7467-7472.

36 M. Noori, A. C. Aragonès, G. Di Palma, N. Darwish, S. W. Bailey, Q. Al-Galiby, I. Grace, D. B. Amabilino, A. González-Campo and I. Díez-Pérez, Sci. Rep., 2016, 6, 37352 .

37 J. M. Soler, E. Artacho, J. D. Gale, A. García, J. Junquera, P. Ordejón and D. Sánchez-Portal, J. Phys.: Condens. Matter, 2002, 14, 2745.

38 N. Troullier and J. L. Martins, Phys. Rev. B: Condens. Matter Mater. Phys., 1991, 43, 1993.

39 T. Ozaki, Phys. Rev. B: Condens. Matter Mater. Phys., 2003, 67, 155108.

40 D. M. Ceperley and B. Alder, Phys. Rev. Lett., 1980, 45, 566. 41 J. Ferrer, C. J. Lambert, V. M. García-Suárez, D. Z. Manrique, D. Visontai, L. Oroszlany, R. Rodríguez-Ferradás, I. Grace, S. Bailey and K. Gillemot, New J. Phys., 2014, 16, 093029. 